\title{
Acute Radiological Abnormalities after Bronchial Thermoplasty: A Prospective Cohort Trial
}

\author{
Julia N.S. d'Hooghe ${ }^{a}$ Inge A.H. van den Berk ${ }^{b}$ Jouke T. Annema ${ }^{a}$ \\ Peter I. Bonta ${ }^{a}$ \\ Departments of a Pulmonology and ${ }^{b}$ Radiology, Academic Medical Center, University of Amsterdam, \\ Amsterdam, The Netherlands
}

\section{Keywords}

Bronchial thermoplasty $\cdot$ Radiology $\cdot$ Asthma

\begin{abstract}
Background: Bronchial thermoplasty (BT) is a novel treatment for severe asthma based on radiofrequency energy delivery to the larger airways. Although impressive radiological abnormalities have been reported, the incidence, pattern, and behavior over time of acute radiological abnormalities following BT are not well established. Objective: To assess the incidence pattern and behavior over time of acute radiological abnormalities following BT. Methods: This is a prospective, observational imaging study of severe asthma patients participating in the TASMA trial. Imaging of the lung (chest X-ray and/or computed tomography [CT]) was performed routinely before and directly after BT, within 6 weeks and at 6 months' follow-up. Results: Thirty-four chest X-rays were performed within $<5 \mathrm{~h}$ following $34 \mathrm{BT}$ procedures in 12 patients. In $91 \%$ of cases, radiological abnormalities were seen, designated as peribronchial consolidations (97\%) and/ or atelectasis (29\%). Ultra-low-dose (ULD) chest CTs were performed following 16 BT procedures showing abnormalities in all. Four different radiological patterns were identi-
\end{abstract}

\section{KARGER}

E-Mail karger@karger.com www.karger.com/res
This article is licensed under the Creative Commons Attribution NonCommercial-NoDerivatives 4.0 International License (CC BYNC-ND) (http://www.karger.com/Services/OpenAccessLicense) tribution of modified material requires written permission. fied: peribronchial consolidations with surrounding ground glass opacities (94\%), atelectasis (38\%), partial bronchial occlusions (63\%), and bronchial dilatations (19\%). No bronchoscopic intervention was needed. At 6 months' follow-up, in a single patient, high-resolution chest $C T$ showed a focal bronchiectasis in a single airway. Conclusions: There is a high incidence of acute radiological abnormalities after BT. Four distinct radiological patterns can be identified on ULD chest CT, which resolve without clinical impact in virtually all cases.

(C) 2017 The Author(s) Published by S. Karger AG, Basel

\section{Introduction}

Bronchial thermoplasty (BT) is a bronchoscopic treatment for patients with severe, refractory asthma that is based on radiofrequency energy delivery to the airways [1]. Impressive radiological abnormalities can occur following BT treatment, including (sub)lobar atelectasis (Fig. 2, 3B; online suppl. Fig. S1; for all online suppl. ma-

J.N.S. d'Hooghe and I.A.H. van den Berk contributed equally to this work.

Peter I. Bonta, $\mathrm{MD}, \mathrm{PhD}$

Department of Pulmonology, F5-144, Academic Medical Center University of Amsterdam

Meibergdreef 9, NL-1105 AZ Amsterdam (The Netherlands) E-Mail p.i.bonta@amc.nl 


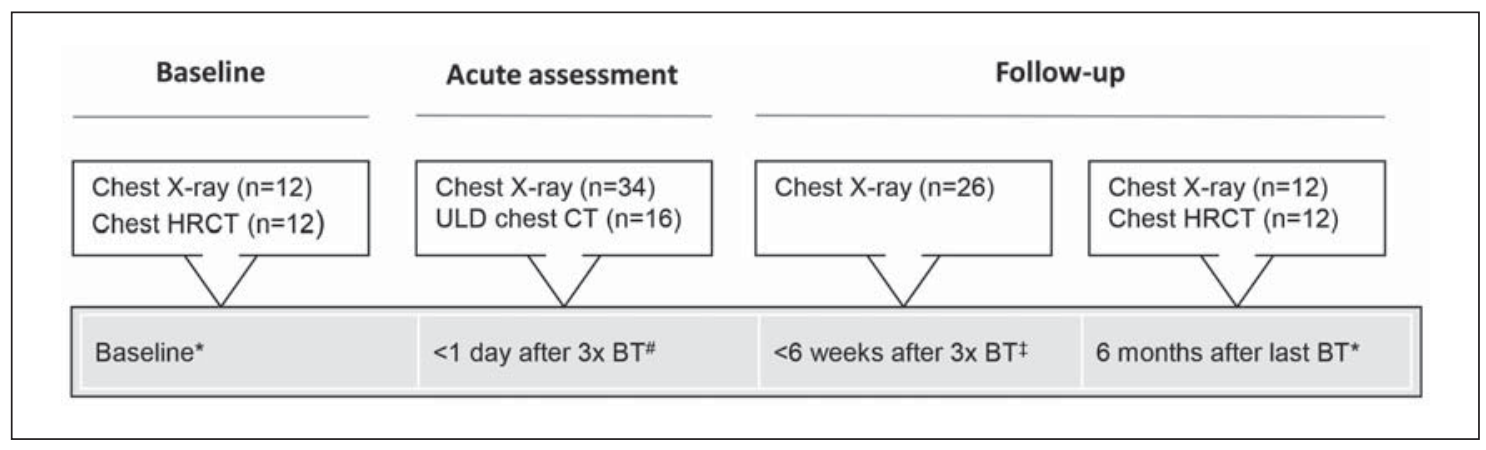

Fig. 1. Diagram of radiological imaging before and after bronchial thermoplasty (BT) ( $n=12$ patients, $n=36 \mathrm{BT}$ procedures). ${ }^{*}$ Chest $\mathrm{X}$-ray and high-resolution computed tomography (HRCT) were performed at baseline and at 6 months after the last BT $(n=12$; all patients). ${ }^{*}$ Chest X-rays were performed within $5 \mathrm{~h}$ after BT $(n=$ $34 / 36$, after 2 BT procedures, only an ultra-low-dose [ULD] chest

terial, see www.karger.com/doi/10.1159/000477586). The aim of this study was to investigate the incidence, pattern, and behavior over time of acute radiological abnormalities following BT.

\section{Methods}

A prospective observational cohort study was performed as part of the TASMA "Unravelling Targets of Therapy in Bronchial Thermoplasty in Severe Asthma" trial (ClinicalTrials.gov No. NCT02225392) from April 2014 to September 2016. The study was approved by the Ethics Committee (NL45394.018.13). All patients provided written informed consent. Patients with severe asthma who fulfilled the World Health Organization (WHO) or modified innovative medicines initiative criteria of severe refractory asthma were included $[2,3]$. Patients were treated with BT according to the current standard during a bronchoscopy with conscious sedation or general anesthesia $[4,5]$. The flow of radiological assessment is summarized in Figure 1. Imaging of the lung (chest X-ray and/or computed tomography [CT]) was performed routinely before and directly after BT, within 6 weeks thereafter, and at 6 months' follow-up. Before and 6 months after BT, high-resolution CT images were obtained with a Philips Brilliance 64-slice CT scanner (Philips, Healthcare, Best, The Netherlands; $1 \mathrm{~mm}$ thickness). Directly after BT, to minimize ionizing radiation exposure, ultra-low-dose (ULD) chest CT images were obtained with the Somatom Force scanner (Siemens, Medical Solution, Forchheim, Germany; $1 \mathrm{~mm}$ thickness). Since at the start of the study the existence of acute radiological abnormalities directly after BT was unknown and the ULD chest CT scanner was not available, in some cases only a chest X-ray was obtained at first. An experienced chest radiologist (I.A.H.B.) evaluated all images and scored the following radiological abnormalities: peribronchial consolidation with/ or without surrounding ground glass opacity, (sub)segmental or (sub)lobar atelectasis, partial bronchial occlusions, bronchial dilatation, and bronchiectasis [6]. An asthma exacerbation was de-

Acute Radiological Abnormalities after

Bronchial Thermoplasty
CT was performed) and ULD chest CT was performed $<24 \mathrm{~h}$ after BT ( $n=16 / 36$, after the first 20 BT procedures, only a chest X-ray was performed since the ULD chest CT scanner was not available at the start of the study). ${ }^{\ddagger}$ Chest X-ray was performed $<6$ weeks after BT $(n=26 / 36)$.

fined as an exacerbation requiring (to increase the dose of) oral or systemic steroids or a doubling dose of inhalation corticosteroids during $>3$ consecutive days. Analyses were performed using SPSS software version 22 (IBM, Armonk, NY, USA).

\section{Results}

Twelve patients underwent $36 \mathrm{BT}$ procedures. All patients used high doses of inhalation corticosteroids/longacting beta-agonists, and $50 \%$ were also treated with oral or systemic steroids. Acute radiological abnormalities were seen after all $36 \mathrm{BT}$ procedures on chest X-ray and/ or ULD chest CT. $91 \%$ of chest X-rays $(n=34)$ after BT showed abnormalities, most commonly peribronchial consolidations (97\%) and/or atelectasis (29\%) (Fig. 2; online suppl. Fig. S1).

All 16 ULD chest CTs performed directly after BT showed acute radiological abnormalities that could be classified into 4 distinct patterns: peribronchial consolidations with surrounding parenchymal ground glass (94\%), atelectasis (38\%), partial bronchial occlusions (63\%), and bronchial dilatations (19\%) (Fig. 3). 31\% of the ULD chest CTs showed ground glass opacities in the neighboring non-BT-treated lobe. These opacities in the non-BT-treated lobe were either in continuum with the BT-treated lobe ( $n=3$, incomplete fissures: $n=2 / 3$ ) or they were located in the periphery of the non-BT-treated lobe with large areas of normal lung parenchyma in between $(n=5)$. No abnormalities were detected in the nonBT-treated right middle lobe. Interestingly, ULD chest CT was able to distinguish BT-treated airways from adja-

Respiration 2017;94:258-262

DOI: $10.1159 / 000477586$ 


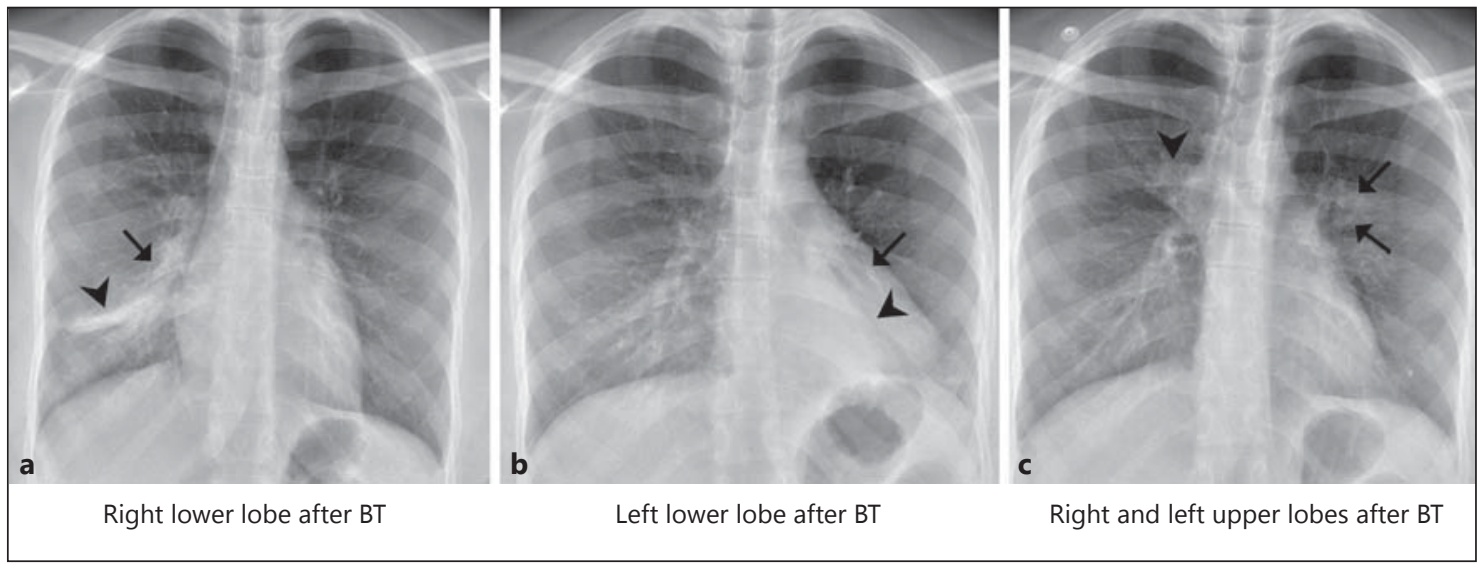

Fig. 2. Acute radiological abnormalities on chest $\mathrm{X}$-ray $<5 \mathrm{~h}$ after bronchial thermoplasty (BT). Chest X-ray (posteroanterior view) $<5 \mathrm{~h}$ after BT of the right lower lobe (a), left lower lobe (b), and both upper lobes (c) showing peribronchial consolidations (arrows) and (sub)segmental atelectasis (arrowheads) in a single patient. Radiological resolution within 6 weeks after BT is shown for both right and left lower lobes (c).
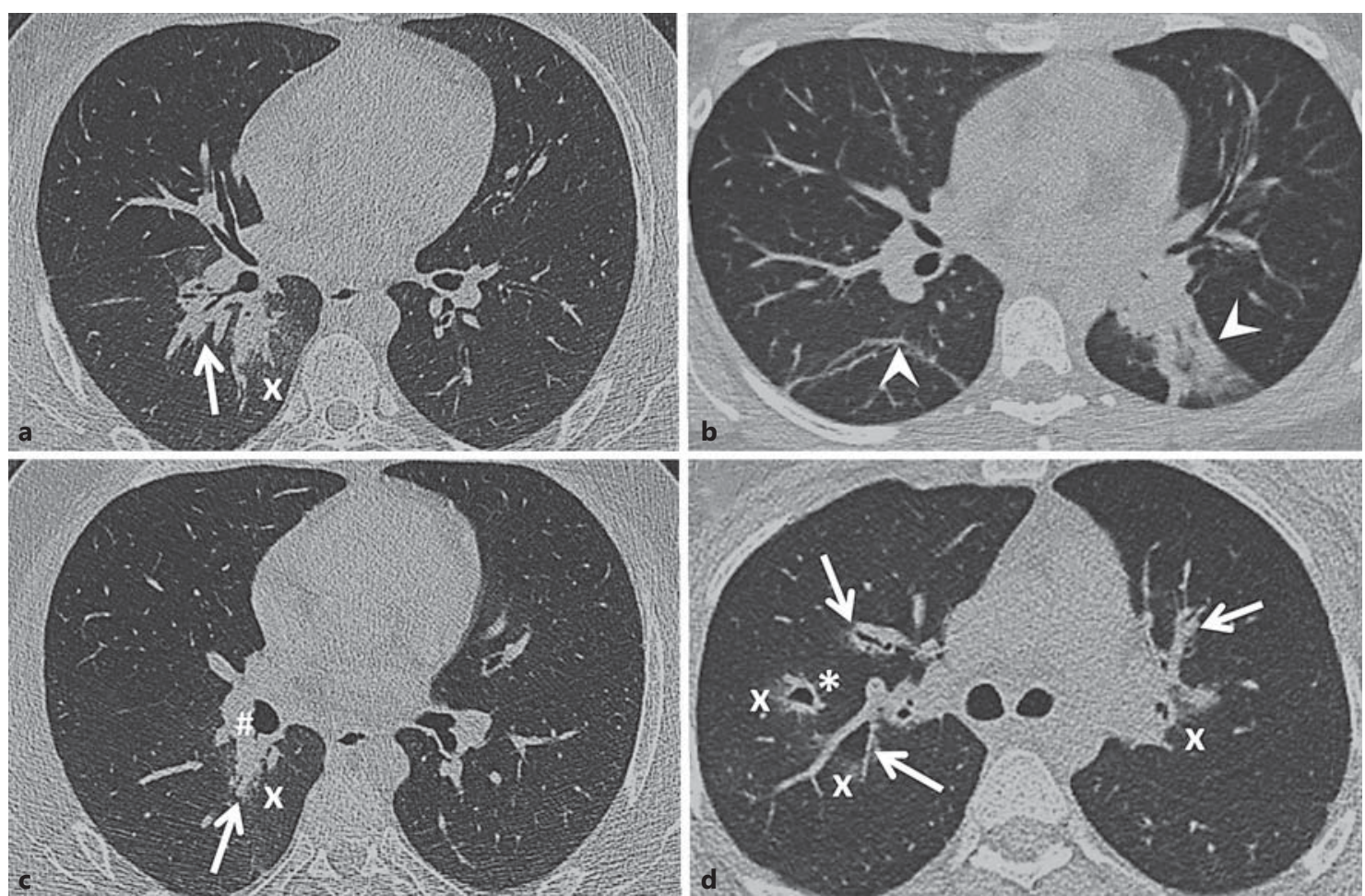

Fig. 3. Four different patterns of acute computed tomography (CT) abnormalities after bronchial thermoplasty (BT): peribronchial consolidations with surrounding parenchymal ground glass opacity, atelectasis, partial bronchial occlusion, and bronchial dilatation. Ultra-low-dose chest CT-detected radiological abnormalities $<24 \mathrm{~h}$ after BT. a Peribronchial consolidation (arrow) with surrounding parenchymal ground glass opacity (cross) in the right lower lobe. No abnormalities were detected in the adjacent right middle lobe. b Subsegmental atelectasis in the apex of the left lower lobe (arrowhead). Three weeks after BT, the right lower lobe shows residual abnormalities compatible with plate atelectasis (arrowhead). c Partial bronchial occlusion of the apical segment (\#) with concomitant peribronchial consolidation (arrow) with surrounding ground glass (cross) of the right lower lobe. $\mathbf{d}$ Focal bronchial dilatation $(\varnothing 0.6 \mathrm{~mm}$; asterisk) in the right upper lobe and peribronchial consolidations (arrows) with surrounding ground glass (crosses) in both upper lobes. 


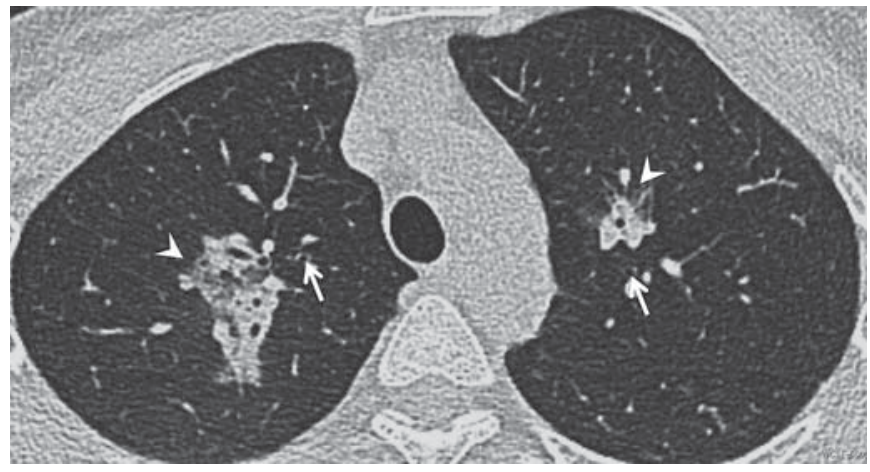

Fig. 4. Airway specificity of bronchial thermoplasty (BT) treatment by radiological abnormalities on ultra-low-dose (ULD) chest computed tomography (CT). ULD chest CT-detected peribronchial consolidations with surrounding ground glass in BT-treated segments of the left and right upper lobes (arrowheads). No abnormalities were detected in the adjacent apical subsegments of both upper lobes that could not be reached by the radiofrequency catheter due to their anatomical position (arrows).

cent airways that were not reached with the radiofrequency catheter due to their anatomical position, most commonly the apical subsegments of the upper lobes (Fig. 4).

Since the presence of radiological abnormalities might be related to postprocedure asthma exacerbations, this association was investigated. In 17 out of 31 (55\%) BT procedures with an abnormal chest X-ray, an asthma exacerbation occurred. However, in 2 out of 3 BT procedures with a normal chest X-ray, an asthma exacerbation was also present. All asthma exacerbations were treated with a course of oral steroids. After 8 procedures, antibiotics were prescribed because of fever or ongoing symptoms without clinical signs of pneumonia. No bronchoscopic intervention was needed.

In patients with radiological abnormalities on chest $\mathrm{X}$-ray, $73 \%$ of chest $\mathrm{X}$-rays showed complete resolution within 6 weeks, and all patients showed complete resolution at 6 months after BT. Follow-up chest high-resolution CT 6 months after the last BT revealed a single case with a focal bronchiectasis without clinical impact.

\section{Discussion}

This study shows that BT causes acute transient radiological lung abnormalities on chest X-ray and/or ULD chest $\mathrm{CT}$ in all patients. ULD chest CT provides superior imaging in comparison to chest X-ray, and 4 different radiological patterns were identified: peribronchial consol- idations with surrounding ground glass, atelectasis, partial bronchial occlusions, and bronchial dilatations. On ULD chest CT, non-BT-treated (adjacent) airways during a BT procedure could be clearly identified from those that were treated. The strengths of this study are its prospective design and the systematic evaluation of the radiological images by an experienced chest radiologist.

Recently, acute radiological abnormalities after BT have been described by Debray et al. [7] in 13 patients, which confirmed our findings of transient radiological abnormalities in all patients in the BT-treated lung lobes the day after the procedure. In line with our results, predominantly peribronchial consolidations with ground glass opacities and partial occlusion/filling of the bronchial lumen were found. Similarly, residual bronchial dilatation was seen in rare cases. In contrast, lobar volume loss/atelectasis was found more often when compared to the present study. Additionally, Debray et al. [7] also found pulmonary opacities in the non-BT-treated middle lobe in 5 cases, which was postulated to be an effect of heat diffusion along the bronchial tree. However, in contrast, data from our study show that airways not reached by BT showed no abnormalities and were clearly distinguishable from those that were treated with BT. We could confirm ground glass opacities in non-BT-treated lobes in up to one-third of cases. In case of abnormalities seen in the non-BT-treated lobe directly adjacent to BT-treated lobes, the mechanism postulated by Debray et al. [7], that comprises the extension of parenchymal edema or inflammation by heat shock, seems a more likely explanation. In case of radiological abnormalities observed in distal areas of the non-BT-treated lobes, we propose that based on radiological characteristics and distribution properties, mucosal blood and/or mucus that runs from the BT-treated upper lobes to the lower non-BT-treated situated areas of the lungs are a plausible explanation.

Acute effects of radiofrequency energy delivery to human airways are expected based on earlier studies. An animal study showed altered airway smooth muscle in canines at 1 week after BT and thickening of the bronchial wall on CT, histologically correlating to inflammatory edema of the airway wall [8]. A case report showed semi-acute bronchial wall thickening on a chest CT scan 7 days after BT [9]. Furthermore, in agreement with radiofrequency ablation of pulmonary neoplasms, the observed acute radiological abnormalities after BT could be explained by the temperature gradient or heat shock [1012]. Indeed, it is described that a large temperature increase causes tissue coagulation and destroys microcirculation, which might induce the peribronchial consolida- 
tions. A moderate temperature increase causes autolysis and microvascular hemorrhage resulting in inflammation and edema resembling ground glass abnormalities [10-12]. The observed ground glass opacities reaching more peripheral of the BT-treated airway as opposed to the consolidations located directly peribronchially fits well with this explanation. To counteract these heat shock effects, periprocedural corticosteroid treatment seems appropriate.

The radiological abnormalities observed immediately after BT treatment reinforce our view that BT certainly modifies the airway. Most probably, the mechanism of action of BT involves a combination of several airway modifications, such as reduction in airway smooth muscle mass and nerves, inflammation and/or microcirculation. A decrease of airway smooth muscle and nerves has recently been reported in biopsies taken before and after BT treatment by Pretolani et al. [13].

In conclusion, there is a high incidence of acute radiological abnormalities after BT. Four distinct radiological patterns can be identified on ULD chest CT, which resolve without clinical impact in virtually all cases.

\section{References}

1 Chung KF, Wenzel SE, Brozek JL, Bush A, Castro M, Sterk PJ, Adcock IM, Bateman ED, Bel EH, Bleecker ER, Boulet LP, Brightling C, Chanez P, Dahlen SE, Djukanovic R, Frey U, Gaga M, Gibson P, Hamid Q, Jajour NN, Mauad T, Sorkness RL, Teague WG: International ERS/ATS guidelines on definition, evaluation and treatment of severe asthma. Eur Respir J 2014;43:343-373.

2 Bel EH, Sousa A, Fleming L, Bush A, Chung KF, Versnel J, Wagener AH, Wagers SS, Sterk $\mathrm{PJ}$, Compton $\mathrm{CH}$; Unbiased Biomarkers for the Prediction of Respiratory Disease Outcome (U-BIOPRED) Consortium, Consensus Generation: Diagnosis and definition of severe refractory asthma: an international consensus statement from the Innovative Medicine Initiative (IMI). Thorax 2011;66:910917.

3 Bousquet J, Mantzouranis E, Cruz AA, AitKhaled N, Baena-Cagnani CE, Bleecker ER, Brightling CE, Burney P, Bush A, Busse WW, Casale TB, Chan-Yeung M, Chen R, Chowdhury B, Chung KF, Dahl R, Drazen JM, Fabbri LM, Holgate ST, Kauffmann F, Haahtela T, Khaltaev N, Kiley JP, Masjedi MR, Mohammad Y, O'Byrne P, Partridge MR, Rabe KF, Togias A, van Weel C, Wenzel
S, Zhong N, Zuberbier T: Uniform definition of asthma severity, control, and exacerbations: Document presented for the world health organization consultation on severe asthma. J Allergy Clin Immunol 2010;126: 926-938.

4 Cox G, Miller JD, McWilliams A, Fitzgerald JM, Lam S: Bronchial thermoplasty for asthma. Am J Respir Crit Care Med 2006;173: 965-969.

5 d'Hooghe J, et al: Propofol and Remifentanil sedation for Brochial Thermoplasty. Respiration 2017, DOI 10.1159/000452478.

6 Hansell DM, Bankier AA, MacMahon H, McLoud TC, Muller NL, Remy J: Fleischner Society: glossary of terms for thoracic imaging. Radiology 2008;246:697-722.

7 Debray MP, Dombret MC, Pretolani M, Thabut G, Alavoine L, Brillet PY, Taille C, Khalil A, Chanez P, Aubier M: Early computed tomography modifications following bronchial thermoplasty in patients with severe asthma. Eur Respir J 2017;49: 1601565.

8 Danek CJ, Lombard CM, Dungworth DL, Cox PG, Miller JD, Biggs MJ, Keast TM, Loomas BE, Wizeman WJ, Hogg JC, Leff AR: Reduction in airway hyperresponsiveness to methacholine by the application of RF energy in dogs. J Appl Physiol 2004;97:1946-1953.

9 McCambridge J, Kruklitis R: Transient bronchial wall thickening after bronchial thermoplasty for asthma. J Bronchol Intervent Pulmonol 2016;23:51-53.

10 Koizumi T, Tsushima K, Tanabe T, Agatsuma T, Yokoyama T, Ito M, Kanda S, Kobayashi T, Yasuo M: Bronchoscopy-guided cooled radiofrequency ablation as a novel intervention therapy for peripheral lung cancer. Respiration 2015;90:47-55.

11 Abtin FG, Eradat J, Gutierrez AJ, Lee C, Fishbein MC, Suh RD: Radiofrequency ablation of lung tumors: imaging features of the postablation zone. Radiographics 2012;32:947-969.

12 Miao Y, Ni Y, Bosmans H, Yu J, Vaninbroukx J, Dymarkowski S, Zhang H, Marchal G: Radiofrequency ablation for eradication of pulmonary tumor in rabbits. J Surg Res 2001;99: 265-271.

13 Pretolani M, Bergqvist A, Thabut G, Dombret MC, Knapp D, Hamidi F, Alavoine L, Taille C, Chanez P, Erjefalt IS, Aubier M: Effectiveness of bronchial thermoplasty in patients with severe refractory asthma: clinical and histopathological correlations. J Allergy Clin Immunol 2017;139:1176-1185. 